\title{
Current Advances in Immuno-oncology for Head and Neck Cancer
}

\author{
${ }^{1}$ Michael-John Devlin, ${ }^{2}$ Martin D Forster
}

\begin{abstract}
Head and neck squamous cell carcinoma (HNSCC) is the 6th most common cancer globally, originating from the epithelial surface of the upper aerodigestive tract from the lips to the larynx. It commonly presents with locally advanced disease, with a recurrence rate of around $50 \%$ despite aggressive multimodality treatment involving surgery, radiotherapy and chemotherapy or EGFR inhibition as appropriate. Improvements in understanding the underlying cancer biology and its evolution within the complex interactions of the tumor microenvironment, there is gathering interest in and evidence for the use of immunomodulating agents in the management of HNSCC. Immune checkpoint inhibitors, primarily programmed cell death protein 1 (PD-1) inhibitors to date, which inhibit the inhibitory interaction between PD-1 and its ligand PD-L1, have demonstrated durable improvements in patient outcomes in advanced/metastatic HNSCC, with both nivolumab and pembrolizumab being granted FDA approval in 2016.
\end{abstract}

There are numerous clinical trials ongoing exploring the role of checkpoint inhibitors both as single agents and in combination, administered with established modalities such as chemotherapy and radiotherapy, as well as alongside other novel immune modulators. These trials are not limited to advanced/ metastatic HNSCC, but also explore neoadjuvant or adjuvant settings. As studies complete and more data become available, immunotherapy agents are likely to have expanding roles within the treatment algorithms of HNSCC, and with greater biomarker development have the potential to further improve patient outcomes via a personalized therapy approach.

Keywords: Cancer immunology, Cancer immunotherapy, Head and neck cancer, Head and neck squamous cell carcinoma, Immune checkpoint inhibitors, SCCHN

How to cite this article: Devlin MJ, Forster MD. Current Advances in Immuno-oncology for Head and Neck Cancer. Int J Head Neck Surg 2018;9(2):78-86.

Source of support: Nil

Conflict of interest: None

\section{INTRODUCTION}

Head and neck cancers encompass malignancies that originate in the paranasal sinuses, nasal cavity, oral

\footnotetext{
${ }^{1,2}$ Professor

1,2 UCL Cancer Institute, University College London, London, UK
}

Corresponding Author: Martin D Forster, Professor, UCL Cancer Institute, University College London, London, UK, e-mail:martin.forster@ucl.ac.uk cavity, pharynx and larynx. With more than 550,000 cases diagnosed worldwide each year and around 300,000 deaths, it is the sixth leading cancer by incidence. ${ }^{1}$

Over $90 \%$ are squamous cell carcinoma (SCCHN), with a male predominance. Certain lifestyle and environmental risk factors, such as alcohol consumption and smoking, have long been established as risk factors for the development of SCCHN with a multiplicative risk for patients who are regularly exposed to both. ${ }^{2}$ More recently human papillomavirus (HPV) type 16 has emerged as a driver for a significant proportion of oropharyngeal squamous cell carcinomas, with more uncertainty over its implication in other sites, and for other subtypes of the virus. ${ }^{3}$. This HPV driven subtype is associated with an epidemiology, local pattern of disease spread and outcome that is quite separate from non-HPV associated disease and as such it is increasingly being regarded as a distinct clinical entity. ${ }^{4-6}$

Despite aggressive primary multimodality treatment involving surgery, radiotherapy, chemotherapy and, where appropriate EGFR inhibition, locally advanced SCCHN has a recurrence rate of $\sim 50 \%$, with very poor outcomes despite subsequent therapies. ${ }^{7}$

\section{IMMUNOLOGY OF HEAD AND NECK SQUAMOUS CELL CARCINOMA}

In order to proliferate locally and metastasize, cancer cells must develop mechanisms that allow them to avoid detection and subsequent elimination by the host immune system. The basis of immunotherapy is the idea that the host immune system can be activated to overcome these acquired mechanisms, recognize the cancer as non-self and eliminate it (Fig. 1).

To achieve an effective antitumor immune response, T-lymphocytes must be able to both infiltrate the tumor and perform their function appropriately ${ }^{8}$ with the frequency of tumor infiltrating lymphocytes (TILs) and their subtypes being linked to prognosis in multiple cancer pathologies. ${ }^{9}$ In SCCHN, an increased TIL presence, ${ }^{10}$ particularly CD3+, CD8+ and FOXP3+ sub-types, ${ }^{11}$ is associated with a more favorable prognosis.

FOXP3+ regulatory T-cells (Tregs) suppress immune response and as such should hinder both detection and elimination of cancer cells ${ }^{12}$ and are associated with a poor prognosis in multiple tumor types. ${ }^{13}$ The positive association observed between infiltration with FOXP3+ 


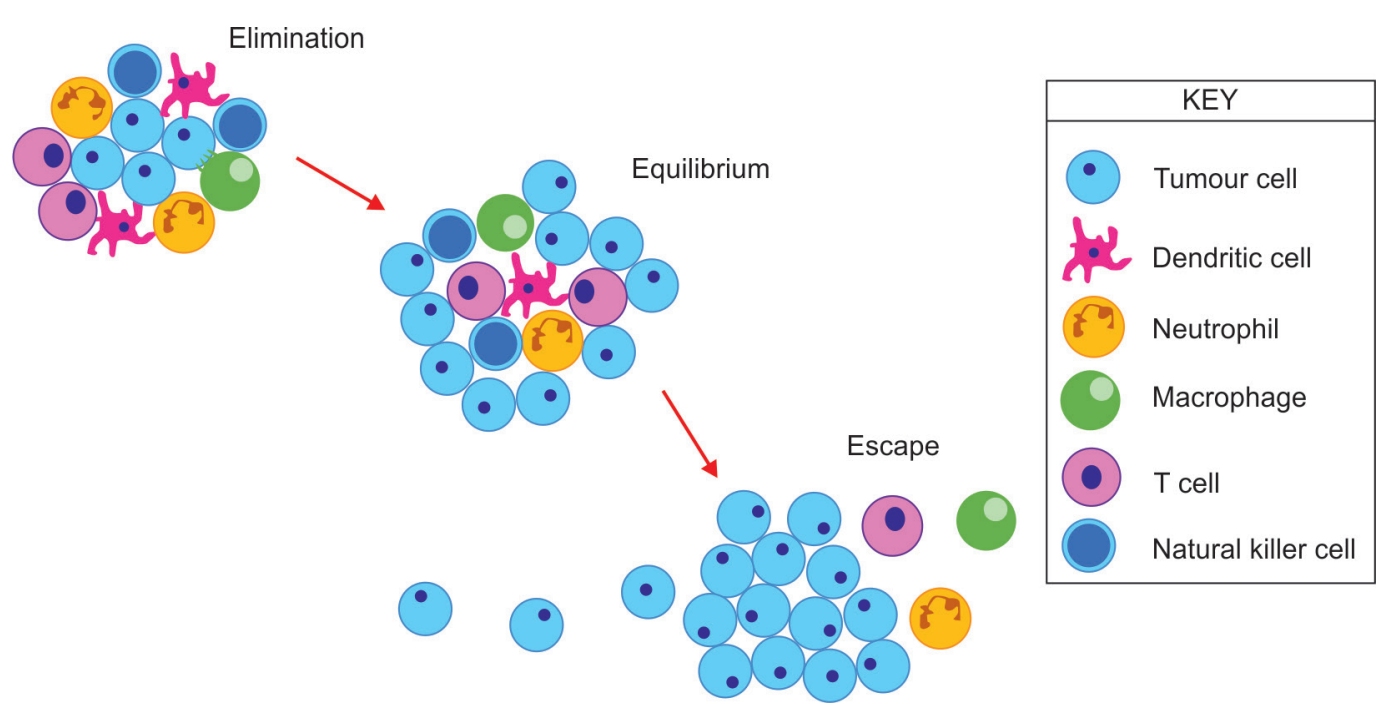

Fig. 1: Immune control and evasion: Interaction between tumor cells and the host immune system within the tumor microenvironment

cells and an improved prognosis in SCCHN seems counter-intuitive but it is also observed in colorectal cancer, epithelial ovarian cancer and lymphoma. ${ }^{14-16}$ The balance between the different TIL clonal populations may influence the effects they have within the tumor microenvironment. Alongside differences in the tumor microenvironments between pathologies, FOXP3+ $\mathrm{T}$ cells exist as three phenotypically and functionally distinct subtypes, ${ }_{17}^{17}$ which may account for this prognostic variation observed across disease sites. In colorectal cancer, it has been found that the FOXP3+lo non-suppressive T cells act in a proinflammatory manner and indicate a better prognosis in than FOXP3+hi cells. ${ }^{18}$ Whether similar findings exist in $\mathrm{SCCHN}$ is an area that requires more research.

Some cancers avoid an immune response by preventing lymphocyte infiltration into the tumor and as such are often referred to as either 'immune-excluded tumors' or 'immune deserts' depending on the immune response around the tumor periphery. ${ }^{19}$

SCCHN does not typically fall into these categories, having one of the more immune-infiltrated cancer types, ${ }^{20}$ suggesting that the tumors use other mechanisms to modulate the immune environment to avoid elimination by the TILs that are present.

This may be achieved through numerous mechanisms such as an increase in the immunosuppressive cytokines IL-10, ${ }^{21}$ IL- $6{ }^{22}$ and TGF-B, ${ }^{23}$ overexpression of antigens causing T-cell tolerance, ${ }^{24}$ deficiency or alterations of tumor human leukocyte antigen (HLA) class I molecules expression ${ }^{25,26}$ and aberrant activation of the transcription factors signal transducers and activators of transcription 3 (STAT3) ${ }^{27}$ and NF-kB. ${ }^{23}$ Better understanding of the key mechanisms involved in the maintenance of an immunosuppressive tumor microenvironment in SCCHN may lead to more effective strategies to overcome them and hence improve disease control and patient outcomes.

\section{Immune Checkpoint Inhibition}

To avoid autoimmunity a series of checkpoints exist on the surface of immune cells, with the activation of a T-cell response being a balance of coinhibitory and costimulatory molecules and their ligands. ${ }^{28}$ By preventing signalling from coinhibitory checkpoints, checkpoint inhibitors aim to generate an enhanced activation of the immune response to tumor cells. In SCCHN, agents that inhibit interaction between, programmed death-1 (PD-1), cytotoxic T-lymphocyte-associated antigen 4 (CTLA-4), or lymphocyte activation gene-3 (LAG-3) and their ligands are currently undergoing evaluation, with other newer agents in development (Fig. 2).

\section{PD-1 and PD-L1 Checkpoint Inhibition}

When PD-1 interacts with its ligand PD-L1, anticancer immunity is suppressed via decreased cytokine production, alongside induction of $\mathrm{T}$ lymphocyte anergy and apoptosis. As such, cancer cells can upregulate PD-L1 in order to functionally inactivate T-cell immune surveillance ${ }^{29,30}$ and PD-L1 overexpression has been reported at between $46-100 \%$ across studies in SCCHN, ${ }^{31}$ the wide range likely owing to a combination of variation in staining techniques and sample preservation.

Nivolumab, an IgG4 PD-1 inhibitor, demonstrated an improvement in both overall survival (OS) and progression free survival (PFS) in the phase III study CheckMate 141.

This study compared nivolumab with the physician's choice of second line single agent (docetaxel, methotrexate or cetuximab) in patients with platinum resistant, recurrent or metastatic HNSCC. A total of 361 patients were recruited and allocated to treatment with a 2:1 randomisation. Whilst response rates (RR) were low in all arms, it was higher in the nivolumab group (13.3\%), with 6 complete responses (CR) and 26 partial responses (PR) reported. 


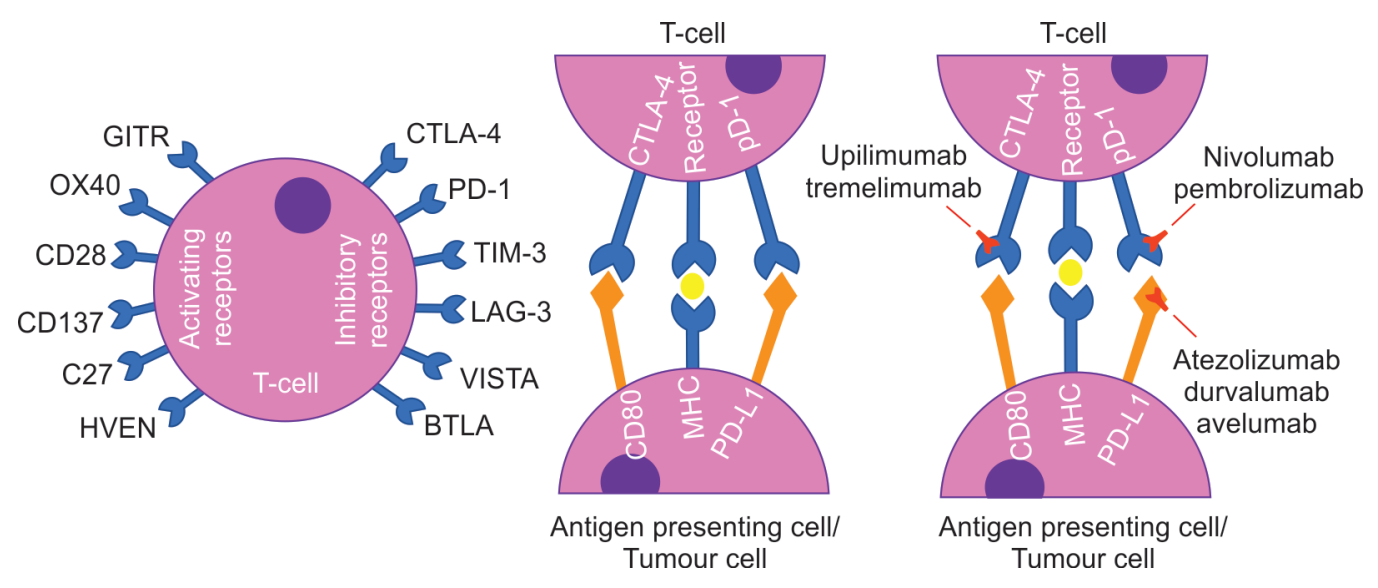

Fig. 2: Immune checkpoints: Coinhibitory and costimulatory checkpoints are potential targets for immunotherapies

In comparison, the RR in the chemotherapy arm was only $5.8 \%$ (including $1 \mathrm{CR}$ ). Patients who received nivolumab had a 30\% lower risk of death (HR 0.70; 95\% CI 0.51-0.96) with an increase in both median overall survival (OS) (7.5 months versus 5.1 months) and estimated landmark progression free survival (PFS) at 6 months (19.7\% versus $9.9 \%$ ). This increased benefit at later time-points gives promise to durable benefit for responding patients, as seen in other tumor types where data are more mature. ${ }^{32}$ In addition to the higher efficacy seen, treatment with nivolumab was found to be more tolerable with a reduction in grade 3 or 4 adverse events $(13.1 \%$ versus $35.1 \%)$ and a relative improvement in patient reported outcomes (PROMS) and other quality of life parameters. ${ }^{33}$

Patterns of response to immunotherapy agents differ from that of standard treatment, with delayed response commonly being observed and less commonly initial enlargement of tumors or the development of new lesions giving the appearance of disease progression and such patients can receive treatment beyond this 'pseudo-progression' $^{32,34}$ The recognition of these unique response characteristics has lead to the development of the immunerelated RECIST criteria. ${ }^{35}$ The outcomes of patients who received treatment beyond progression as part of CheckMate 141 were presented at the European Society of Medical Oncology 2017 Annual Congress (ESMO 2017). Of those who progressed, 62 patients $(42 \%)$ received at least one further dose of nivolumab and $15 \mathrm{had}$ a subsequent reduction in target lesion size with 3 achieving a reduction of greater than $30 \%$, with no increase in grade 3 or 4 toxicities, ${ }^{36}$ suggesting that treatment beyond progression may be considered for patients where there appears to be some clinical benefit observed and it is felt safe to do so.

Pembrolizumab is another IgG4 PD-1 antibody, which has also been explored in the treatment of patients with recurrent or metastatic SCCHN. KEYNOTE-012 was a phase Ib study that included an expansion cohort of 132 SCCHN patients. This trial reported a RR of $18 \%$ at 9 months, which included 4 CR and 20 PR, with a median
OS of 8 months and a 6-month PFS of $23 \%$. Overall the treatment was felt to be very tolerable with grade 3 or 4 toxicity was reported in $9 \%$ of patients. ${ }^{37}$ These data led to the accelerated approval of pembrolizumab by the FDA for the treatment of SCCHN. Early outcomes from the confirmatory phase III trial, KEYNOTE-040, were presented at ESMO 2017 and AACR 2018 $8^{38,39}$ although the final published manuscript is awaited. This study compared pembrolizumab with standard therapies in a similar design to CheckMate 141 study, although with a complex statistical analysis plan. It demonstrated a median OS of 8.4 months with pembrolizumab compared to 7.1 months in the control arm, with a hazard ratio of 0.80 (95\% confidence interval 0.66-0.99), reaching statistically significance $(p=0.016)$ with the updated data from the full population. It is also likely that the OS data may have been confounded by some patients within the control arm (12\%) going on to receive immune checkpoint inhibitors post progression (cross-over effect).

The benefit of other agents that interrupt the interaction between PD-1 and PD-L1 in SCCHN, such as durvalumab, atezolizumab and avelumab, is currently being investigated in ongoing trials.

\section{Double Checkpoint Inhibition}

The upregulation of alternative immune checkpoints may cause resistance to anti-PD-1/PD-L1 treatment ${ }^{40}$ and has driven the rationale for combining treatments to target more than one co-inhibitory molecule. The use of combination checkpoint inhibitors (CPI) has been successful in improving both response rates and survival in other cancer pathologies, with several combinations under ongoing investigation in SCCHN.

CTLA-4 is expressed on the surface of activated T cells, where it can prevent the co-stimulatory interaction of B7 and $\mathrm{CD} 28$, subsequently down regulating $\mathrm{T}$ cell proliferation and IL-2 production. Inhibiting CTLA-4 results in an increase in T-Cell activation alongside maintenance of high-frequency T-cell receptor clonotypes. ${ }^{41,42}$ 
The combination of CTLA-4 and PD-L1 inhibition has resulted in higher response rates than monotherapy alone in metastatic melanoma, renal cell cancer and small cell lung cancer. ${ }^{4-45}$ In SCCHN, this combination is being evaluated in a number of ongoing trials both in the first line and metastatic settings with results eagerly awaited.

LAG-3 is expressed on the surface of activated CD4+ and CD8+ T-cells and certain subtypes of natural killer and dendritic cells. It suppresses both activation and proliferation of $\mathrm{T}$ cells and assists in Treg suppressive function, ${ }^{46-50}$ promoting a immunosuppressive immune microenvironment. BMS-986016 is an anti-LAG-3 antibody that is being explored both as monotherapy in combination in a phase I/IIa dose expansion and escalation study (NCT01968109) that involves an SCCHN cohort.

\section{Checkpoint Inhibition and Chemo Radiotherapy}

Chemo Radiotherapy (CRT) can trigger immunogenic cell death creating a cascade of endogenous molecules called "damage-associated molecular patterns" (DAMPs) which recruit and stimulate antigen presenting cells, eventually resulting in an adaptive immune response. ${ }^{51}$ This is particularly relevant in SCCHN where a significant proportion of patients will receive (chemo/bio) radiotherapy at some point in their primary management.

In SCCHN, CRT increases the number of CD8+ T effector cells, CD4+ regulatory cells and T cells expressing PD-1, TIM3 and LAG3 within the tumor microenvironment, particularly in oropharyngeal cancers. ${ }^{52}$ An increase in CD3+ and CD8+ TILs has also been shown to positively correlate with clinical outcome to definitive CRT in SCCHN. ${ }^{53}$

The addition of checkpoint blockade following platinum-based CRT has been shown to be feasible, safe and demonstrated an improvement in PFS for patients with non-small cell lung cancer, as demonstrated by the phase III PACIFIC study. ${ }^{54}$

Preclinical data suggest benefits may be better if the CPI are delivered concurrently with the radiotherapy. ${ }^{55}$

There are a number of studies underway investigating the benefits of adding checkpoint inhibition to CRT in the management of SCCHN. The preliminary results of a safety study combining pembrolizumab with cisplatinbased CRT in locally advanced SCCHN was presented at ASCO 2017. Pembrolizumab was administered 4-7 days prior to CRT, three weekly for the duration of CRT and then a further five doses following completion. In total 27 patients were enrolled, of which $74 \%$ had HPV positive oropharyngeal tumors. All subjects received their planned XRT dose, $85 \%$ received target cisplatin dose and $78 \%$ completing the planned doses of pembrolizumab. The addition of pembrolizumab was not felt to have significantly increased treatment toxicity, however three patients required treatment-discontinuation due to immune related adverse events (G2 peripheral motor neuropathy, G3 AST elevation and G1 Lhermitte-like syndrome). The study has now reopened expansion cohorts in $34 \mathrm{HPV}$ positive and $23 \mathrm{HPV}$ negative patients to further evaluate efficacy. ${ }^{56}$

Cetuximab can be administered as an alternative to cisplatin alongside RT for radical treatment of locally advanced SCCHN. It influences both natural killer cell response and dendritic cell maturation ${ }^{57,58}$ and increases expression of inhibitory checkpoints PD-1, TIM-3 and CTLA- 4 on TILs. ${ }^{59,60}$ While a number of trials are exploring the addition of checkpoint blockade to cetuximab-RT, the combination with avelumab is of particular interest due to the propensity of both avelumab and cetuximab to activate antibody-dependent cellular cytotoxicity (ADCC). ${ }^{58,61}$

\section{Other Combination Approaches}

Inhibiting immune checkpoints is only one approach to promoting an anti-cancer immune environment and a number of other agents are being explored in combination with anti-PD-1 and anti-PD-L1 agents to augment their effectiveness.

STAT3 signaling has been implicated in the proliferation, invasion and survival of many cancers, including SCCHN, through upregulation of inhibitory cytokines. ${ }^{62}$ AZD9150 is an oligonucleotide antisense molecule that prevents the production of STAT3, with preclinical evidence of antitumor activity in lymphoma and lung cancer models. ${ }^{63}$ Preclinical studies in SCCHN models have also shown that STAT3 inhibition sensitizes to both chemotherapy and radiotherapy, particularly in nasopharyngeal cancer. ${ }^{64,65}$ AZD9150 is being administered in combination with Durvalumab, a PD-L1 inhibtior, as part of the phase Ib/II SCORES trial for patients with advanced or metastatic SCCHN (NCT02499328). Initial results were announced at the ESMO Congress 2017. Of the 35 patients included in this report, 15 had received prior treatment with an anti-PD-L1 agent. In the CPI naïve arm, a 25\% objective RR was reported, with a $45 \%$ DCR at 12 weeks and $30 \%$ of patients remaining on treatment at 25 weeks. For anti-PD-L1 pretreated patients, 1 complete response and 1 unconfirmed response were reported, with a $20 \%$ DCR at 12 weeks.

Overall the combination was felt safe and deliverable with G3/4 thrombocytopenia and increases in liver enzymes reported for $3.4 \%$ patients, and two treatmentrelated discontinuations (unspecified). These early data are promising, and mature results are eagerly awaited.

CXC Chemokine receptor 2 (CXCR2) is a G proteincoupled receptor for a number of cytokines and is 
implicated in disease proliferation via IL-8 signaling. It is known to be frequently overexpressed in SCCHN. AZD5069 is a novel selective antagonist of CXCR2 and is being given in combination with Durvalumab as a separate arm of the SCORES trial, with results awaited. ${ }^{66,67}$

Indoleamine 2,3-dioxygenase 1 (IDO1) is a catabolizing enzyme that suppresses T-cells and promotes immune suppression and has been associated with poor outcome in laryngeal squamous cell carcinoma. ${ }^{68}$ Epacadostat is an oral inhibitor of IDO1 which is being administered concurrently with pembrolizumab was part of the phase I/II KEYNOTE-037 study for patients with recurrent/metastatic SCCHN. Preliminary results were presented at ASCO 2017 with 36 of 38 patients being efficacy-evaluable at the initial data cut off. The ORR was reported at $31 \%$ with DCR of $58 \%$, regardless of the number of previous lines of treatment. Overall the treatment was tolerated well with fatigue ( $24 \%)$, nausea (11\%) and decreased weight $(11 \%)$ being the most commonly reported adverse events. These promising data have led to plans for a phase III study. ${ }^{69}$

Oncolytic viruses have been found to reduce tumor burden and stimulate antigen presentation preclinically, ${ }^{70,71}$ and when administered concurrently, may help overcome anti-PD-1 resistance by broadening neoantigendirected T-cell responses. ${ }^{72}$

Granulocyte macrophage colony-stimulating factor (GM-CSF) promotes the attraction and maturation of dendritic cells and amplifies antigen presentation. ${ }^{73}$ One of the more advanced oncolytic viruses in development is the locally administered Talimogene Laherparepvec (T-VEC), a modified, live, attenuated herpes simplex virus type 1 . T-VEC has been shown to preferentially infect cancer cells in patients with melanoma, ${ }^{74}$ where it has gained a license after demonstrating improved DRR and mOS. ${ }^{75,76}$ It is being administered alongside Pembrolizumab as part of the phase Ib/III KEYNOTE137 study for patients with recurrent metastatic SCCHN (NCT02626000).

ADXS11-001 is an immunotherapeutic based on live, attenuated listeria monocytogenes, which secretes HPV-E7 tumor antigen as a truncated fusion protein resulting in HPV-specific T-cell generation. ${ }^{77}$ Inhibition of PD-L1 increased ADXS11-011 activity preclinically ${ }^{78}$ and is being administered both as a monotherapy and in conjunction with Durvalumab for the treatment of HPV positive HNSCC as part of a phase I/II study (NCT02291055).

\section{Biomarkers}

Both CheckMate-141 and KEYNOTE-12 explored the impact of HPV positivity as a predictor of response. In Checkmate-141, OS appeared to be longer with nivolumab than chemotherapy regardless of p16 status, however the benefit appeared more pronounced in patients with p16-positive tumors (mOS 9.1 months versus 4.4 months respectively), than in the p16-negative tumors (mOS 7.5 months versus 5.8 months). ${ }^{33}$ KEYNOTE-012 reported better outcomes to pembrolizumab in the HPV-positive patients compared to those that were HPV negative (RR $32 \%$ vs $14 \%$; 6 month PFS $37 \%$ vs $20 \%$ and 6 -month OS $70 \%$ vs $56 \%)^{37}$ again suggesting that the viral-associated SCCHN might gain greater benefit from checkpoint inhibition.

In the survival analysis of CheckMate-141, PD-L1 expression $>1 \%$ was associated with a hazard ratio for death of 0.55 (95\% CI 0.36-0.83) when treated with nivolumab compared to standard therapy. Where PD-L1 expression was $<1 \%$, this HR was increased at $0.89(95 \%$ CI 0.54-1.45). ${ }^{33}$ Similarly, KEYNOTE-012 described PD-L1 expression $>1 \%$ or $<1 \%$ and found that patients with PD-L1 positive tumors had a RR to pembrolizumab of $22 \%$ compared to $4 \%$ in negative tumors, and median OS of 303 days versus 151 days, respectively, ${ }^{37}$ Interestingly, both studies examined PD-L1 positivity on tumor cells alone, however overexpression on immune cells has been linked to response to CPI in metastatic urotherlial cancer ${ }^{79}$ and the combination of expression on both tumor and/or immune cells is being explored in HNSCC within the KEYNOTE-040 study.

Of the 61 patients in KEYNOTE-012 with PD-L1 positive disease, 43 had RNA expression profiling, evaluated with multigene expression signatures derived from patients with melanoma. Of these signatures, a 6-gene INF- $\gamma$ was the top-performing, with significant associations to OR $(p=0.005)$ and PFS $(<0.001)$. On evaluation of the individual signature genes, INF- $\gamma$ inducible MHC-II expression was felt to be the key biological link. Using an optimal cutoff for INF- $\gamma$, positive predictive value for response was $40 \%$ with a negative predictive value of $95 \%$; AUC $=0.8$ (95\% CI 0.61-0.95). This information may assist in identifying patients who are most likely (or least likely) to gain clinical benefit from anti-PD-1 therapy, ${ }^{80}$ either alone or in combination with PD-L1 data.

A high mutational burden or neoantigen load, is associated with response to checkpoint blockade across a number of disease sites ${ }^{81,82}$ with mutational burden decreasing with successful anti-PD-1 treatment ${ }^{83}$ suggesting that selection against mutant neoepitopes may be a critical mechanism of action of this class of drug. The somatic mutational load (ML) and INF- $\gamma$ gene expression profile were both found to be independently predictive of response to pembrolizumab in the 73 patients within KEYNOTE-012 who were HPV and Epstein-Barr Virus (EBV) negative, with ML and INF- $\gamma$ gene expression profile being significantly associated with OR $(p=0.064$ 
and $p=0.001$; AUROC 0.82 and 0.74, respectively). INF- $\gamma$ gene expression profile also remained a significant predictor in HPV and EBV positive patients showing promise as a biomarker of response regardless of viral status. ${ }^{84}$

Several other potential biomarkers are currently under exploration including the epigenetic modification of genes associated with homologous recombination, such as RAD51 and XRCC3, which are thought to alter checkpoint expression ${ }^{85}$ and the identification of different subtypes of SCCHN, each with a distinct microenvironment, ${ }^{86}$ but whether these predict for OS or response to immunotherapy has yet to be established.

\section{CONCLUSION}

Immunotherapy agents have shown promising results in the treatment of HNSCC, with the 2016 FDA approval of both pembrolizumab and nivolumab offering new hope for patients with this disease. As the results of ongoing trials are made available, checkpoint inhibitors in particular may find new roles in the neoadjuvant or adjuvant settings, either as monotherapy or in conjunction with other agents. Along with a deepening appreciation of the tumor-host interaction and careful biomarker selection we are entering an era of personalized immunotherapy treatment, which should translate to improved outcomes for patients with squamous cell carcinoma of the head and neck.

\section{REFERENCES}

1. Jemal A, Bray F, Center MM, Ferlay J, Ward E, Forman D. Global cancer statistics. CA Cancer J Clin, 2011. 61(2):69-90.

2. Blot WJ, McLaughlin JK, Winn DM, Austin DF, Greenberg RS, Preston-Martin S, et al. Smoking and drinking in relation to oral and pharyngeal cancer. Cancer Res, 1988;48(11):3282-3287.

3. Mork J, Lie AK, Glattre E, Clark S, Hallmans G, Jellum E, et al. Human papillomavirus infection as a risk factor for squamous-cell carcinoma of the head and neck. N Engl J Med, 2001. 344(15):1125-1131.

4. Ang KK, Harris J, Wheeler R, Weber R, Rosenthal DI, NguyenTân PF, et al. Human papillomavirus and survival of patients with oropharyngeal cancer. N Engl J Med, 2010. 363(1):24-35.

5. Weinberger PM, Yu Z, Haffty BG, Kowalski D, Harigopal M, Brandsma J, et al. Molecular classification identifies a subset of human papillomavirus--associated oropharyngeal cancers with favorable prognosis. J Clin Oncol, 2006. 24(5): p. 736-747.

6. Cantrell SC, Peck BW, Li G, Wei Q, Sturgis EM, Ginsberg LE. Differences in imaging characteristics of HPV-positive and HPV-Negative oropharyngeal cancers: a blinded matched-pair analysis. AJNR Am J Neuroradiol, 2013;34(10):2005-2009.

7. Argiris A, Karamouzis MV, Raben D, Ferris RL. Head and neck cancer. The Lancet. 2008 May 17;371(9625):1695-1709.

8. Lanitis E, Dangaj D, Irving M, Coukos G. Mechanisms regulating T-cell infiltration and activity in solid tumors. Annals of Oncology. 2017 Sep 21;28(suppl_12):xii18-32.

9. Gooden MJ, de Bock GH, Leffers N, Daemen T, Nijman HW. The prognostic influence of tumour-infiltrating lymphocytes in cancer: a systematic review with meta-analysis. British journal of cancer. 2011 Jun;105(1):93-103.

10. Xu Q, Wang C, Yuan X, Feng Z, Han Z. Prognostic value of tumor-infiltrating lymphocytes for patients with head and neck squamous cell carcinoma. Translational oncology. 2017 Feb 1;10(1):10-16.

11. de Ruiter EJ, Ooft ML, Devriese LA, Willems SM. The prognostic role of tumor infiltrating T-lymphocytes in squamous cell carcinoma of the head and neck: a systematic review and meta-analysis. Oncoimmunology. 2017 Nov 2;6(11):e1356148.

12. Miyara M, Sakaguchi S. Natural regulatory T cells: mechanisms of suppression. Trends in molecular medicine. 2007 Mar 1;13(3):108-116.

13. Kost SE, Kakal JA, Nelson BH. The prognostic value of FoxP3+ tumor-infiltrating lymphocytes in cancer: a critical review of the literature. Clinical cancer research. 2012 Jun 1;18(11):3022-3029.

14. Milne K, Köbel M, Kalloger SE, Barnes RO, Gao D, Gilks CB, et al. Systematic analysis of immune infiltrates in high-grade serous ovarian cancer reveals CD20, FoxP3 and TIA- 1 as positive prognostic factors. PloS one. 2009 Jul 29;4(7):e6412.

15. Salama P, Phillips M, Grieu F, Morris M, Zeps N, Joseph D, et al. Tumor-infiltrating FOXP3+ T regulatory cells show strong prognostic significance in colorectal cancer. Journal of clinical oncology. 2009 Jan 10;27(2):186-192.

16. Carreras J, Lopez-Guillermo A, Fox BC, Colomo L, Martinez A, Roncador $\mathrm{G}$, et al. High numbers of tumor-infiltrating FOXP3positive regulatory $\mathrm{T}$ cells are associated with improved overall survival in follicular lymphoma. Blood, 2006;108(9): 2957-2964.

17. Miyara M, Yoshioka Y, Kitoh A, Shima T, Wing K, Niwa A, et al. Functional delineation and differentiation dynamics of human CD4+ T cells expressing the FoxP3 transcription factor. Immunity. 2009 Jun 19;30(6):899-911.

18. Saito T, Nishikawa H, Wada H, Nagano $Y$, Sugiyama D, Atarashi K, et al. Two FOXP3+ CD4+ T cell subpopulations distinctly control the prognosis of colorectal cancers. Nature medicine. 2016 Jun;22(6):679.

19. Kim JM, Chen DS. Immune escape to PD-L1/PD-1 blockade: seven steps to success (or failure). Annals of Oncology. 2016 May 20;27(8):1492-1504.

20. Mandal R, Şenbabaoğlu Y, Desrichard A, HavelJJ, Dalin MG, Riaz $\mathrm{N}$, et al. The head and neck cancer immune landscape and its immunotherapeutic implications. JCI insight. 2016 Oct 20;1(17).

21. Jebreel A, Mistry D, Loke D, Dunn G, Hough V, Oliver K, et al. Investigation of interleukin 10,12 and 18 levels in patients with head and neck cancer. The Journal of Laryngology \& Otology. 2007 Mar;121(3):246-252.

22. Choudhary MM, France TJ, Teknos TN, Kumar P. Interleukin-6 role in head and neck squamous cell carcinoma progression. World J Otorhinolaryngol Head Neck Surg, 2016;2(2):90-97.

23. Freudlsperger C, Bian Y, Wise SC, Burnett J, Coupar J, Yang $X$, et al. TGF- $\beta$ and NF- $\mathrm{KB}$ signal pathway cross-talk is mediated through TAK1 and SMAD7 in a subset of head and neck cancers. Oncogene. 2013 Mar;32(12):1549.

24. Ferris RL, Immunology and Immunotherapy of Head and Neck Cancer. J Clin Oncol, 2015;33(29):3293-3304.

25. Ferris RL, Hunt JL, Ferrone S. Human leukocyte antigen (HLA) class I defects in head and neck cancer. Immunologic research. 2005 Nov 1;33(2):113-133.

26. López-Albaitero A, Nayak JV, Ogino T, Machandia A, Gooding $\mathrm{W}$, DeLeo AB, et al. Role of antigen-processing machinery 
in the in vitro resistance of squamous cell carcinoma of the head and neck cells to recognition by CTL. The Journal of Immunology. 2006 Mar 15;176(6):3402-3409.

27. Mali SB. Review of STAT3 (Signal Transducers and Activators of Transcription) in head and neck cancer. Oral Oncol, 2015. 51(6):565-569.

28. Yao S, Zhu Y, Chen L. Advances in targeting cell surface signalling molecules for immune modulation. Nat Rev Drug Discov, 2013. 12(2):130-146.

29. Riley JL. PD-1 signaling in primary $T$ cells. Immunol Rev, 2009;229(1):114-125.

30. Gordon SR, Maute RL, Dulken BW, Hutter G, George BM, McCracken MN, et al. PD-1 expression by tumour-associated macrophages inhibits phagocytosis and tumour immunity. Nature, 2017;545(7655):495-499.

31. Zandberg DP, Strome SE. The role of the PD-L1: PD-1 pathway in squamous cell carcinoma of the head and neck. Oral oncology. 2014 Jul 1;50(7):627-632.

32. Topalian SL, Sznol M, McDermott DF, Kluger HM, Carvajal $\mathrm{RD}$, Sharfman WH, et al. Survival, durable tumor remission, and long-term safety in patients with advanced melanoma receiving nivolumab. J Clin Oncol, 2014;32(10):1020-1030.

33. Ferris RL, Blumenschein Jr G, Fayette J, Guigay J, Colevas AD, et al. Nivolumab for recurrent squamous-cell carcinoma of the head and neck. New England Journal of Medicine. 2016 Nov 10;375(19):1856-1867.

34. George S, Motzer RJ, Hammers HJ, Redman BG, Kuzel TM, Tykodi SS, et al. Safety and efficacy of nivolumab in patients with metastatic renal cell carcinoma treated beyond progression: a subgroup analysis of a randomized clinical trial. JAMA oncology. 2016 Sep 1;2(9):1179-1186.

35. Seymour L, Bogaerts J, Perrone A, Ford R, Schwartz LH, Mandrekar S, et al. iRECIST: guidelines for response criteria for use in trials testing immunotherapeutics. The Lancet Oncology. 2017 Mar 1;18(3):e143-152.

36. Haddad RB, Blumenschein G, Fayette J, Guigay J, Colevas $\mathrm{AD}$, Licitra $\mathrm{L}$, et al. Treatment beyond progression with nivolumab in patients with recurrent or metastatic (R/M) squamous cell carcinoma of the head and neck (SCCHN) in the phase 3 checkmate 141 study: A biomarker analysis and updated clinical outcomes. Annals of Oncology. 2017 Sep 1;28 (suppl_5).

37. Chow LQ, Haddad R, Gupta S, Mahipal A, Mehra R, Tahara $\mathrm{M}$, et al. Antitumor activity of pembrolizumab in biomarkerunselected patients with recurrent and/or metastatic head and neck squamous cell carcinoma: results from the phase $\mathrm{Ib}$ KEYNOTE-012 expansion cohort. Journal of Clinical Oncology. 2016 Sep 19;34(32):3838-3845.

38. Cohen EE, Soulieres D, Le Tourneau C, Dinis J, Licitra LF, Ahn MJ, et al. Health-related quality of life (HRQoL) of pembrolizumab (pembro) vs standard of care (SOC) for recurrent/metastatic head and neck squamous cell carcinoma (R/M HNSCC) in KEYNOTE-040. Annals of Oncology, 2017;28(5).

39. Soulieres D. Updated survival results of the KEYNOTE-040 study of pembrolizumab vs standard-of-care chemotherapy for recurrent or metastatic head and neck squamous cell carcinoma. in AACR. 2018 Chicago, USA.

40. Koyama S, Akbay EA, Li YY, Herter-Sprie GS, Buczkowski KA, Richards WG, et al. Adaptive resistance to therapeutic PD-1 blockade is associated with upregulation of alternative immune checkpoints. Nat Commun, 2016;7:10501.
41. Snyder A, Makarov V, Merghoub T, Yuan J, Zaretsky JM, Desrichard A, et al. Genetic basis for clinical response to CTLA-4 blockade in melanoma. New England Journal of Medicine. 2014 Dec 4;371(23):2189-2199.

42. Blank CU, Enk A. Therapeutic use of anti-CTLA-4 antibodies. International immunology. $2014 \mathrm{Jul}$ 18;27(1):3-10.

43. Larkin J, Chiarion-Sileni V, Gonzalez R, Grob JJ, Cowey CL, Lao $\mathrm{CD}$, et al. Combined nivolumab and ipilimumab or monotherapy in untreated melanoma. New England journal of medicine. $2015 \mathrm{Jul}$ 2;373(1):23-34.

44. Antonia SJ, López-Martin JA, Bendell J, Ott PA, Taylor M, Eder JP, et al. Nivolumab alone and nivolumab plus ipilimumab in recurrent small-cell lung cancer (CheckMate 032): a multicentre, open-label, phase 1/2 trial. The Lancet Oncology. 2016 Jul 1;17(7):883-895.

45. Hammers HJ, Plimack ER, Infante JR, Rini BI, McDermott DF, Lewis LD, et al. Safety and efficacy of nivolumab in combination with ipilimumab in metastatic renal cell carcinoma: the CheckMate 016 study. J Clin Oncol. 2017 Dec 1;35(34):3851-3858.

46. Triebel F, Jitsukawa S, Baixeras E, Roman-Roman SE, Genevee C, Viegas-Pequignot EV, et al. LAG-3, a novel lymphocyte activation gene closely related to CD4. Journal of Experimental Medicine. 1990 May 1;171(5):1393-1405.

47. Workman CJ, Vignali DA. Negative regulation of T cell homeostasis by lymphocyte activation gene-3 (CD223). J Immunol, 2005;174(2):688-695.

48. Workman CJ, Wang Y, El Kasmi KC, Pardoll DM, Murray PJ, Drake CG, et al. LAG-3 regulates plasmacytoid dendritic cell homeostasis. The Journal of Immunology. 2009 Feb 15;182(4):1885-1891.

49. Camisaschi C, Casati C, Rini F, Perego M, De Filippo A, Triebel F, et al. LAG-3 expression defines a subset of CD4+ CD25highFoxp3+ regulatory $\mathrm{T}$ cells that are expanded at tumor sites. The Journal of Immunology. 2010 Jun 1;184(11):6545-6551.

50. Huang CT, Workman CJ, Flies D, Pan X, Marson AL, Zhou $\mathrm{G}$, et al. Role of LAG-3 in regulatory T cells. Immunity. 2004 Oct 1;21(4):503-513.

51. Bezu L, Gomes-da-Silva LC, Dewitte H, Breckpot K, Fucikova J, Spisek R, et al. Combinatorial strategies for the induction of immunogenic cell death. Frontiers in immunology. 2015 Apr $24 ; 6: 187$.

52. Sridharan V, Margalit DN, Lynch SA, Severgnini M, Zhou J, Chau NG, et al. Definitive chemoradiation alters the immunologic landscape and immune checkpoints in head and neck cancer. British journal of cancer. 2016 Jul;115(2):252.

53. Balermpas P, Michel Y, Wagenblast J, Seitz O, Weiss C, Rödel $\mathrm{F}$, et al. Tumour-infiltrating lymphocytes predict response to definitive chemoradiotherapy in head and neck cancer. British journal of cancer. 2014 Jan;110(2):501.

54. Antonia SJ, Villegas A, Daniel D, Vicente D, Murakami S, Hui $\mathrm{R}$, et al. Durvalumab after chemoradiotherapy in stage III non-small-cell lung cancer. New England Journal of Medicine. 2017 Nov 16;377(20):1919-1929.

55. Dovedi SJ, Adlard AL, Lipowska-Bhalla G, McKenna C, Jones S, Cheadle EJ, et al. Acquired resistance to fractionated radiotherapy can be overcome by concurrent PD-L1 blockade. Cancer research. 2014 Oct 1;74(19):5458-5468.

56. Powell SF, Gitau MM, Sumey CJ, Reynolds JT, Lohr M, McGraw S, et al. Safety of pembrolizumab with chemoradiation (CRT) in locally advanced squamous cell carcinoma of the head and neck (LA-SCCHN). 
57. Srivastava RM, Lee SC, Andrade Filho PA, Lord CA, Jie HB, Davidson $\mathrm{HC}$, et al. Cetuximab-activated natural killer and dendritic cells collaborate to trigger tumor antigen-specific T-cell immunity in head and neck cancer patients. Clinical Cancer Research. 2013 Apr 1;19(7):1858-1872.

58. Pahl JH, Ruslan SE, Buddingh EP, Santos SJ, Szuhai K, Serra $\mathrm{M}$, et al. Anti-EGFR antibody cetuximab enhances the cytolytic activity of natural killer cells toward osteosarcoma. Clinical cancer research. 2012 Jan 15;18(2):432-441.

59. Jie HB, Schuler PJ, Lee SC, Srivastava RM, Argiris A, Ferrone $\mathrm{S}$, et al. CTLA-4+ regulatory T cells increased in cetuximabtreated head and neck cancer patients suppress NK cell cytotoxicity and correlate with poor prognosis. Cancer research. 2015 Jun 1;75(11):2200-2210.

60. Jie HB, Srivastava RM, Argiris A, Bauman JE, Kane LP, Ferris RL. Increased PD-1+ and TIM-3+ TILs during cetuximab therapy inversely correlate with response in head and neck cancer patients. Cancer immunology research. 2017 May 1;5(5):408-416.

61. Boyerinas B, Jochems C, Fantini M, Heery CR, Gulley JL, Tsang KY, et al. Antibody-dependent cellular cytotoxicity activity of a novel anti-PD-L1 antibody avelumab (MSB0010718C) on human tumor cells. Cancer immunology research. 2015 Oct 1;3(10):1148-1157.

62. Johnston PA, Sen M, Hua Y, Camarco D, Shun TY, Lazo JS, et al. High-content pSTAT3/1 imaging assays to screen for selective inhibitors of STAT3 pathway activation in head and neck cancer cell lines. Assay and drug development technologies. 2014 Feb 1;12(1):55-79.

63. Hong D, Kurzrock R, Kim Y, Woessner R, Younes A, Nemunaitis J, et al. AZD9150, a next-generation antisense oligonucleotide inhibitor of STAT3 with early evidence of clinical activity in lymphoma and lung cancer. Science translational medicine. 2015 Nov 18;7(314):314ra185.

64. Pan Y, Zhou F, Zhang R, Claret FX. Stat3 inhibitor Stattic exhibits potent antitumor activity and induces chemo-and radio-sensitivity in nasopharyngeal carcinoma. PloS one. 2013 Jan 29;8(1):e54565.

65. Adachi M, Cui C, Dodge CT, Bhayani MK, Lai SY. Targeting STAT3 inhibits growth and enhances radiosensitivity in head and neck squamous cell carcinoma. Oral oncology. 2012 Dec 1;48(12):1220-1226.

66. Nicholls DJ, Wiley K, Dainty I, MacIntosh F, Phillips C, Gaw A, et al. Pharmacological characterization of AZD5069, a slowly reversible CXC chemokine receptor 2 antagonist. Journal of Pharmacology and Experimental Therapeutics. 2015 May 1;353(2):340-350.

67. Chan LP, Wang LF, Chiang FY, Lee KW, Kuo PL, Liang CH. IL-8 promotes HNSCC progression on CXCR1/2-meidated NOD1/RIP2 signaling pathway. Oncotarget. 2016 Sep 20;7(38):61820.

68. Ye J, Liu H, Hu Y, Li P, Zhang G, Li Y. Tumoral indoleamine 2, 3-dioxygenase expression predicts poor outcome in laryngeal squamous cell carcinoma. Virchows Archiv. 2013 Jan 1;462(1):73-81.

69. Hamid O, Bauer TM, Spira AI, Olszanski AJ, Patel SP, Wasser JS, et al. Epacadostat plus pembrolizumab in patients with SCCHN: Preliminary phase I/II results from ECHO-202/ KEYNOTE-037.

70. Prestwich RJ, Errington F, Ilett EJ, Morgan RS, Scott KJ, Kottke T,et al. Tumor infection by oncolytic reovirus primes adaptive antitumor immunity. Clinical Cancer Research. 2008 Nov 15;14(22):7358-7366.
71. Woller N, Knocke S, Mundt B, Gürlevik E, Strüver N, Kloos A, et al. Virus-induced tumor inflammation facilitates effective DC cancer immunotherapy in a Treg-dependent manner in mice. The Journal of clinical investigation. 2011 Jul 1;121(7):2570-2582.

72. Woller N, Gürlevik E, Fleischmann-Mundt B, Schumacher A, Knocke S, Kloos AM, et al. Viral infection of tumors overcomes resistance to PD-1-immunotherapy by broadening neoantigenome-directed T-cell responses. Molecular Therapy. 2015 Oct 1;23(10):1630-1640.

73. Kaufman HL, Ruby CE, Hughes T, Slingluff CL. Current status of granulocyte-macrophage colony-stimulating factor in the immunotherapy of melanoma. Journal for immunotherapy of cancer. 2014 Dec;2(1):11.

74. Johnson DB, Puzanov I, Kelley MC. Talimogene laherparepvec (T-VEC) for the treatment of advanced melanoma. Immunotherapy. 2015 Jul;7(6):611-619.

75. Greig SL. Talimogene laherparepvec: first global approval. Drugs. 2016 Jan 1;76(1):147-154.

76. Andtbacka RH, Kaufman HL, Collichio F, Amatruda T, Senzer $\mathrm{N}$, Chesney J, et al. Talimogene laherparepvec improves durable response rate in patients with advanced melanoma. J clin Oncol. 2015 Sep 1;33(25):2780-2788.

77. Miles B, Safran HP, Monk BJ. Therapeutic options for treatment of human papillomavirus-associated cancers-novel immunologic vaccines: ADXS11-001. Gynecologic oncology research and practice. 2017 Dec;4(1):10.

78. Cohen EE, Moore KN, Slomovitz BM, Chung CH, Anderson ML, Morris SR, et al. Phase I/II study of ADXS11-001 or MEDI4736 immunotherapies alone and in combination, in patients with recurrent/metastatic cervical or human papillomavirus (HPV)-positive head and neck cancer. Journal for immunotherapy of cancer. 2015 Nov 4;3(Suppl 2).

79. Mariathasan S, Turley SJ, Nickles D, Castiglioni A, Yuen K, Wang Y, et al. TGF attenuates tumour response to PD-L1 blockade by contributing to exclusion of T cells. Nature. 2018 Feb;554(7693):544.

80. Seiwert TY, Burtness B, Weiss J, Eder JP, Yearley J, Murphy E, Nebozhyn M, McClanahan T, Ayers M, Lunceford JK, Mehra R. Inflamed-phenotype gene expression signatures to predict benefit from the anti-PD-1 antibody pembrolizumab in PD-L1+ head and neck cancer patients. J Clin Oncol, 2015:33(15).

81. Hugo W, Zaretsky JM, Sun L, Song C, Moreno BH, Hu-Lieskovan S, et al. Genomic and transcriptomic features of response to anti-PD-1 therapy in metastatic melanoma. Cell. 2016 Mar 24;165(1):35-44.

82. Rizvi NA, Hellmann MD, Snyder A, Kvistborg P, Makarov V, Havel JJ, Lee W, Yuan J, Wong P, Ho TS, Miller ML. Mutational landscape determines sensitivity to PD-1 blockade in non-small cell lung cancer. Science. 2015 Apr 3;348(6230): 124-128.

83. Riaz N, Havel JJ, Makarov V, Desrichard A, Urba WJ, Sims JS, et al. Tumor and microenvironment evolution during immunotherapy with nivolumab. Cell. 2017 Nov 2;171(4): 934-949.

84. Haddad RI, Seiwert TY, Chow LQ, Gupta S, Weiss J, Gluck I, et al. Genomic determinants of response to pembrolizumab in head and neck squamous cell carcinoma (HNSCC). J Clin Oncol, 2017:35(15).

85. Rieke DT, Ochsenreither S, Klinghammer K, Seiwert TY, Klauschen F, Tinhofer I, et al. Methylation of RAD51B, XRCC3 
and other homologous recombination genes is associated with expression of immune checkpoints and an inflammatory signature in squamous cell carcinoma of the head and neck, lung and cervix. Oncotarget. 2016 Nov 15;7(46):75379.
86. Keck MK, Zuo Z, Khattri A, Stricker TP, Brown CD, Imanguli $\mathrm{M}$, et al. Integrative analysis of head and neck cancer identifies two biologically distinct HPV and three non-HPV subtypes. Clinical cancer research. 2015 Feb 15;21(4):870-881. 